\title{
Association between Non-Alcoholic Fatty Liver Disease and Carotid Artery Intima-Media Thickness on B-Mode Ultrasonogram
}

Fahmida Shams*1, Golam Azam*2, Akanda Fazle Rabbi ${ }^{3}$, Mizanur Rahman ${ }^{4}$

\begin{abstract}
:
Background: Prevalence of non-alcoholic fatty liver disease (NAFLD) is rapidly increasing worldwide. It is closely associated with abdominal obesity, dyslipidemia, hypertension, and type 2 diabetes, which are all features of the metabolic syndrome. Increased carotid intima media thickness (CIMT) is generally accepted as an early indicator of atherosclerosis and has been related to cardiovascular risk factors, and cardiovascular disease including incidence of myocardial infarction and stroke. The increasing rate of type II diabetes mellitus, abdominal obesity, sedentary life style and changes in the dietary habit, all are leading to serious health burden like NAFLD in Bangladesh. These patients have a higher risk of cardiovascular diseases. Early prediction of such incidence may help reduce the deadly consequences of NAFLD.
\end{abstract}

Methods: This cross sectional study was carried out at the department of Radiology and Imaging of Dhaka Medical College and Hospital during the period of July 2015 to June 2017. Study population comprised of the outpatients who underwent abdominal ultrasonogram. Measurement of CIMT was done by high resolution real-time B-mode ultrasonogram at the same time. Body weight, height, blood pressure and available biochemical test reports were recorded along with a brief clinical history. Data were analyzed by SPSS version 23.

Results: A total of 101 subjects were included in this study. Of them, on ultrasonographic examination of abdomen 49(48.5\%) had normal liver assigned as group A and 52(51.5\%) patients had fatty liver disease assigned as group B. Mean age of group A and group B patients were $36.8 \pm 12.4$ and $46.15 \pm 10.3$ years, and male:female ratio was $20: 29$ and $28: 24$ respectively. Family history of cardiovascular disease, diabetes mellitus and current smoking history were $14.3 \%$ versus $19.2 \%, 24.5 \%$ versus $44.2 \%$ and $14.3 \%$ versus $11.5 \%$ respectively among group $A$ and group B. Frequency of NAFLD was grade I fatty liver $32.6 \%$, grade II fatty liver $38.4 \%$ and grade I II fatty liver was $28.8 \%$. Mean BMI was $24.8 \pm 4.1$ versus $27.9 \pm 3.5(p<0.001)$ and serum total cholesterol $(\mathrm{mg} / \mathrm{dl})$ was $175.1 \pm 41.3$ versus $207.3 \pm 52.6(P=0.030)$ among two groups. Both these parameters were significant. On the other hand mean systolic blood pressure $(\mathrm{mm} \mathrm{Hg})$ $127.5 \pm 16.1$ versus $127.5 \pm 16.1$ ( $P=0.836)$, mean diastolic blood pressure ( $\mathrm{mm} \mathrm{Hg}$ ) 81.6 \pm 11.6 versus $82.4 \pm 8.7$ $(P=0.7 .8)$, serum triglyceride level $(\mathrm{mg} / \mathrm{dl}) 175.3 \pm 106.1$ versus $213.4 \pm 167.4(P=0.404)$ were not significant among groups. Mean CIMT (mm) was $0.62 \pm 0.15$ in group $A$ and $0.77 \pm 0.17$ in group $B(P<0.001)$ respectively. This result is highly significant. Accuracy of the CIMT in patients with or without NAFLD by ROC curve showed the area under curve (AUC) was 0.752 which was fair outcome of the study. CIMT cut-off at $0.680 \mathrm{~mm}$ showed the highest sensitivity (75\%) and specificity (63\%) for the presence of fatty liver disease by ultrasonogram.

Conclusion: This study was aimed to explore the association between non-alcoholic fatty liver disease and carotid artery intima-media thickness on B-mode ultrasonogram. There is a positive correlation between NAFLD with CIMT. High BMI and raised serum cholesterol has significant role in the development of NAFLD. A large scale study is recommended to find more accurate cut off of CIMT in the relationship with NAFLD.

Keywords: Fatty liver disease, metabolic syndrome, cardiovascular disease, stroke.

\section{Introduction}

Diagnosis of non-alcoholic fatty liver disease (NAFLD), a cause of progress towards the end stage of liver disease, is increasing. ${ }_{-1}^{-}$It represents a spectrum of clinico-pathological conditions that is determined with macrovesicular steatosis in the absence of alcohol consumption. The disease includes clinical, laboratory and pathological conditions ranged from mild steatosis to liver diseases such as non- alcoholic steatohepatitis (NASH), fibrosis, cirrhosis and eventually hepatocellular carcinoma. ${ }^{1,2,3}$ With increasing urbanization and behavioral changes such as decreased physical activity, fat-high-energy diet and increased occurrence of diabetes mellitus type 2, prevalence of NAFLD has increased in the Asian region. ${ }^{1,2,3,4}$ Prevalence of the disease was estimated between 7 and 40 percent in different populations, and in a country like Japan it has been 3-20 folds within the past 20 years. ${ }^{5}$

Most patients with non-cirrhotic NAFLD are asymptomatic in the beginning with incidental detection of raised liver enzymes or fatty liver on ultrasound. Some patients are detected to have fatty liver on ultrasound and raised enzymes during work-up for dyspeptic symptoms, malaise or fatigability, or work-up for other illness. The diagnosis of fatty liver is usually made on ultrasound with exclusion of other causes of fatty liver and raised liver enzymes if present. Ultrasound is an accurate, reliable imaging technique for the detection of fatty liver, as compared with histology, with a sensitivity of $84.8 \%$ and a specificity of $93.6 \%$ for detecting 
around $20-30 \%$ steatosis. $^{6}$ Computed tomography scan and magnetic resonance imaging (MRI) really do not add much and are as good as ultrasound for detecting fat in the liver. ${ }^{6}$ Magnetic resonance spectroscopy is better in detecting fat but none of these modalities can detect the degree of inflammation and fibrosis, hence are not good in differentiating between only steatosis and histological NASH. Since ultrasound is easily available, inexpensive without any radiation risk, it should be the first modality to assess the presence and grading of hepatic steatosis and for the severity of liver disease. On abdominal ultrasound, the radiologist should look for the liver echogenicity and its comparison with that of kidney and spleen, vascular blurring and deep attenuation of ultrasound signal. ${ }^{7}$

NAFLD is commonly associated with visceral obesity, dyslipidemia, insulin resistance, and type 2 diabetes and may represent another component of the metabolic syndrome $(\mathrm{MetS})^{1,8,9}$ a condition associated with a high cardiovascular risk and, in particular, an increased prevalence of carotid lesions. ${ }^{10}$ It is important to determine whether NAFLD is an independent predictor of cardiovascular morbidity and mortality and several studies have suggested that there is an association between NAFLD and cardiovascular disease. . $^{10,11,12,13,14,15}$

Noninvasively detected increased carotid intima media thickness (CIMT) is generally accepted as an early indicator of generalized atherosclerosis and has been related to cardiovascular risk factors, and cardiovascular disease including incidence of myocardial infarction and stroke. ${ }^{16,17,18}$ Some case-controlled and cross-sectional, ${ }^{10,11,12}$ showed a relationship between NAFLD and CIMT. ${ }^{15,19}$ Indeed, it is hypothesized that NAFLD is not merely a marker of cardiovascular disease but may also be involved in its pathogenesis. ${ }^{12}$ A possible relationship between NAFLD and carotid lesions might have important practical consequences,

1. Dr. Fahmida Shams MD (Radiology), Radiologist, Kurmitola General Hospital, Dhaka, Bangladesh.

2. Dr. Md. Golam Azam, Associate Professor, Department of Gastrointestinal, Hepatobiliary and Pancreatic Disorders (GHPD), BIRDEM General Hospital, Shahbag, Dhaka, Bangladesh.

3. Dr. Akanda Fazle Rabbi Associate Professor, Department of Radiology and Imaging, Dhaka Medical College \& Hospital, Dhaka.

4. Prof. Dr. Md. Mizanur Rahman, Dhaka Medical College \& Hospital, Dhaka.

*Fahmida Shams and Golam Azam had equal contributions and will be considered as principal authors.

*Corresponding Author:

Golam Azam

MBBS, MD (Hepatology)

Associate Professor, Department of Gastrointestinal, Hepatobiliary and Pancreatic Disorders (GHPD), BIRDEM General Hospital, Shahbag, Dhaka, Bangladesh.

E mail: drgolamazam@gmail.com considering the frequent incidental finding of hepatic steatosis in subjects undergoing abdominal ultrasound (US) for any reason. In these subjects, a B-mode US assessment of carotid arteries might also be advisable. However, some degree of variability about the mean CIMT values has been observed among all the published reports that result in a difficult evaluation of the magnitude of the observation. For instance, among the different studies, mean CIMT values in NAFLD patients range from $0.64 \pm 0.10 \mathrm{~mm}$ to $1.24 \pm 0.13 \mathrm{~mm} .^{11,19}$ It seems to be important to decide whether further recommendations with regard to carotid atherosclerosis screening should be implemented in all NAFLD patients, as currently available epidemiological data indicate that a value of CIMT at or above $1 \mathrm{~mm}$ at any age is associated with a significantly increased risk of myocardial infarction and/or cerebrovascular disease. ${ }^{18}$ The increasing rate of type II diabetes mellitus, abdominal obesity, sedentary life style and changes in the dietary habit, all are leading to serious health burden like NAFLD in Bangladesh. These patients have a higher risk of cardiovascular diseases. Early prediction of such incidence may help reduce the deadly consequences of NAFLD.

This cross sectional study was carried out in a group of outpatients undergoing abdominal US to establish the strength of the increased CIMT in NAFLD patients. It was conducted at the Department of Radiology and Imaging of Dhaka Medical College and Hospital, Dhaka, during the period of July 2015 to June 2017.

\section{Materials and methods}

This study was a cross sectional study carried out at the department of Radiology and Imaging of Dhaka Medical College and Hospital, Dhaka during the period of July 2015 to June 2017. Adult patients attending for abdominal ultrasonography were selected for this study. NAFLD diagnosis was based on abdominal US and exclusion of other known etiologic factors of chronic liver disease (daily alcohol consumption $\geq 30 \mathrm{~g}$ for men and $\geq 20 \mathrm{~g}$ for women, viral hepatitis, autoimmune hepatitis, and use of hepato-toxic drugs). The control group, were recruited from apparently healthy subjects with normal liver US. Those who had evidence of endocrine or syndromal disorders of obesity, systemic disease or acute illness. After explaining the study objectives, informed written consent was taken. Structured questionnaire was filled up regarding the clinical history and laboratory findings already in hand. B-mode ultrasonogram was carried out for all cases, which was supervised by expert supervisor. The examination was performed using a $3.5 \mathrm{MHz}$ convex transducer (Aloka, Japan). The normal liver parenchyma has a homogeneous echotexture with echogenicity equal to or slightly greater than that of the renal cortex and spleen. When there is fatty infiltration, the liver showed echogenicity higher than the renal cortex and spleen. ${ }^{20}$ Various (0-3) grades of steatosis had been proposed based on visual analysis of the intensity of the echogenicity, provided that the gain setting is optimum. When the echogenicity was just increased, it was grade I; when the echogenic liver obscured the echogenic walls of portal vein branches, it was 
grade II, and, when the echogenic liver obscured the diaphragmatic outline, it was grade III fatty infiltration. ${ }^{21}$ The common carotid arteries were scanned bilaterally using a high-resolution real-time B-mode ultrasonography with a 5-13 MHz linear array transducer (Aloka, Japan). All subjects were examined in supine position with their head turned 45 degrees from the site which was scanned. Measurements were made manually on still images magnified to standard size on-line. The IMT was defined as the distance between the leading edge of the lumen-intima echo and the leading edge of the media-adventitia echo. The common carotid artery IMT was measured on-line in the posterior wall 10-20 mm proximal to the carotid bifurcation in a region free of focal plaque. Three measurements were made on each side, and the values were averaged to produce a mean IMT for each side. The mean Carotid intima-media thickness (C-IMT) was defined as the mean of the right and left IMT of the common carotid artery. ${ }^{22}$ Body mass index classification (WHO criteria): a) If BMI is less than 18.5, it falls within the underweight range. b) If $\mathrm{BMI}$ is 18.5 to $<25$, it falls within the normal. c) If BMI is 25.0 to $<30$, it falls within the overweight range. d) If BMI is 30.0 or higher, it falls within the obese range.

Data were analyzed by computer with the help of SPSS (Statistical Package for Social Sciences) version 23. The data were expressed as frequencies or as means \pm standard deviation. Statistical analyses were done by using appropriate statistical tool like 'chi-square' test, student's 't' test where applicable. Statistical significance was set at 0.05 level and confidence interval at $95 \%$ level. Receiver operating characteristic (ROC) curve were constituted to measure the validity of tests where necessary. Data were presented in the form of table and graphs. Descriptive statistics was presented with frequency table. Association was illustrated with cross tables and test statistics were added in the foot note of the table. Bar and pie charts were generated to illustrate descriptive statistics. The protocol was approved by ethical review committee of Dhaka medical college.

\section{Results:}

A prospective, cross-sectional study was carried out to explore the association between non-alcoholic fatty liver disease and carotid artery intima-media thickness on B-mode ultrasonogram. Non probability (purposive) sampling was done. A total of 101 patients who fulfilled the inclusion/exclusion criteria were enrolled. The results of the study are presented in following tables and figures.

Table I shows demographic profile of the study subjects with or without NAFLD on ultrasonogram $(n=101)$ It shows a significant difference of age among normal liver and NAFLD group. However, gender, family history of CVD, family history of DM and current smokimg habit had no significance

Table I

\begin{tabular}{clll}
\hline & $\begin{array}{l}\text { Ultrasonographic } \\
\text { normal liver (Group A) } \\
(\mathbf{n = 4 9 )}\end{array}$ & $\begin{array}{l}\text { Ultrasonographic } \\
\text { NAFLD (Group B) } \\
(\mathbf{n = 5 2 )}\end{array}$ & p-value \\
\hline $\begin{array}{l}\text { Age (Yrs) Mean } \pm \text { SD } \\
\text { Gender n (\%) }\end{array}$ & $36.8 \pm 12.4$ & $46.15 \pm 10.3$ & - \\
$\quad \begin{array}{l}\text { Male } \\
\text { Female }\end{array}$ & $20(40.8)$ & $28(53.8)$ & 0.233 \\
Family history of CVD n (\%) & $29(59.2)$ & $24(46.2)$ & \\
Yes & $7(14.3)$ & $10(19.2)$ & 0.599 \\
$\quad$ No & $42(85.7)$ & $42(80.8)$ & 0.059 \\
Family history of DM n (\%) & $12(24.5)$ & $23(44.2)$ & \\
Yes & $37(75.5)$ & $29(55.8)$ & 0.771 \\
No & & $6(11.5)$ & \\
Current smoking habit n (\%) & $7(14.3)$ & $46(88.5)$ & \\
Yes & $42(85.7)$ &
\end{tabular}


Bangladesh Crit Care J September 2020; 8 (2): 112-119

Table II shows clinical, anthropometric and laboratory findings of the study subjects with or without NAFLD. It shows that there was significant difference among two groups in considering BMI and serum cholesterol level. Systolic blood pressure, diastolic blood pressure, Fasting blood sugar and serum triglyceride levels were not significant among groups.

Table II

\begin{tabular}{lccc}
\hline & $\begin{array}{c}\text { Ultrasonographic } \\
\text { normal liver group } \\
(\mathrm{n}=49)\end{array}$ & $\begin{array}{c}\text { Ultrasonographic } \\
\text { NAFLD group } \\
(\mathrm{n}=52)\end{array}$ & p-value \\
\hline BMI & $24.8 \pm 4.1$ & $27.9 \pm 3.5$ & $<0.001$ \\
Systolic BP (mm Hg) & $127.5 \pm 16.1$ & $127.5 \pm 16.1$ & 0.836 \\
Diastolic BP (mm Hg) & $81.6 \pm 11.6$ & $82.4 \pm 8.7$ & 0.708 \\
Fasting blood sugar (mmol/L) & $7.6 \pm 3.7$ & $8.8 \pm 3.2$ & 0.202 \\
Total cholesterol (mg/dL) & $175.1 \pm 41.3$ & $207.3 \pm 52.6$ & 0.030 \\
Triglyceride (mg/dL) & $175.3 \pm 106.1$ & $213.4 \pm 167.4$ & 0.404 \\
\hline
\end{tabular}

Fig I shows grading of NAFLD by B-mode ultrasonogram

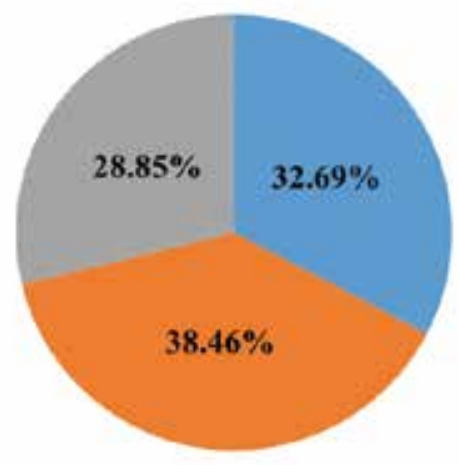

In Gr I Fatty liver

= Gr II Fatty liver

in Gr III Fatty liver

Fig I

Fig II shows grading of NAFLD by B-mode ultrasonogram among different age groups

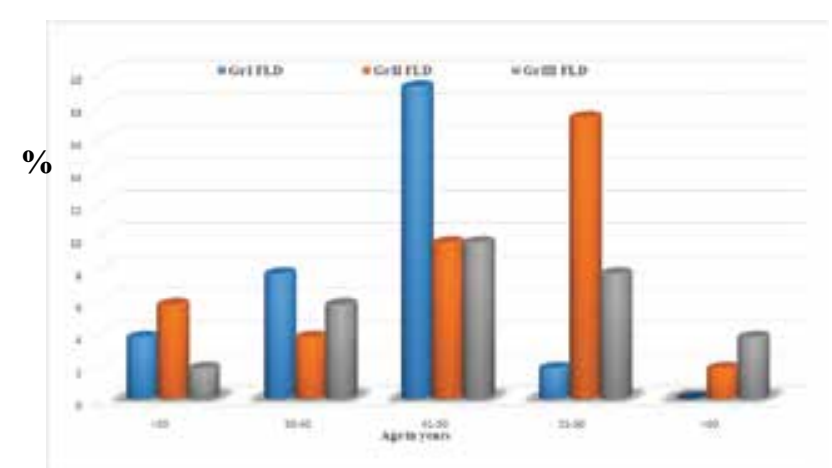

Fig II
Fig III shows mean CIMT in mm among normal liver and different grades of fatty liver disease

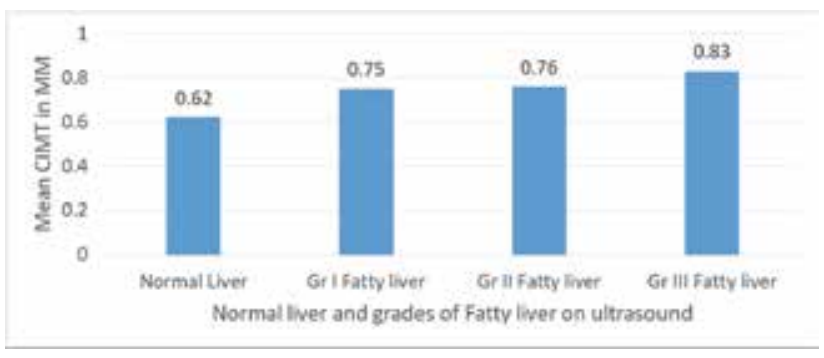

Fig III

Table III shows comparison of mean CIMT among two groups $(\mathrm{n}=101)$. There was a significant difference among the two groups.

Table III

\begin{tabular}{cccc}
\hline $\begin{array}{c}\text { Ultrasonographic } \\
\text { normal liver group } \\
(\mathbf{n}=\mathbf{4 9})\end{array}$ & $\begin{array}{c}\text { Ultrasonographic } \\
\text { NAFLD } \\
\text { group }(\mathbf{n}=\mathbf{5 2})\end{array}$ & p-value \\
\hline CIMT $(\mathrm{mm})$ & $0.62 \pm 0.15$ & $0.77 \pm 0.17$ & $<0.001$ \\
\hline
\end{tabular}

Table IV shows correlation between NAFLD and CIMT and the result was significant.

Table IV

\begin{tabular}{lcc}
\hline & r value & p value \\
\hline NAFLD & 0.429 & $<0.001$ \\
CIMT & & \\
\hline
\end{tabular}


Table V shows ultrasonic comparison of mean CIMT among two groups in respect to gender $(n=101)$ and in both gender, normal liver group have significantly lower CIMT.

Table V

\begin{tabular}{|c|c|c|c|}
\hline & $\begin{array}{l}\text { Ultrasonographic } \\
\text { normal liver group } \\
(n=49)\end{array}$ & $\begin{array}{l}\text { Jltrasonographic } \\
\text { NAFLD } \\
\text { group }(n=52)\end{array}$ & p-value \\
\hline \multicolumn{4}{|c|}{ CIMT $(\mathrm{mm})$ in male } \\
\hline $\mathrm{n}(\%)$ & $20(41.6)$ & $28(58.4)$ & 0.001 \\
\hline mean \pm SD & $0.63 \pm 0.14$ & $0.81 \pm 0.18$ & \\
\hline \multicolumn{4}{|c|}{ CIMT $(\mathrm{mm})$ in female } \\
\hline $\mathrm{n}(\%)$ & $29(54.7)$ & $24(45.3)$ & 0.010 \\
\hline mean $\pm \mathrm{SD}$ & $0.61 \pm 0.15$ & $0.73 \pm 0.16$ & \\
\hline
\end{tabular}

Table VI shows sensitivity, specificity, positive predictive value and negative predictive value at different cut-off points of CIMT for diagnosing NAFLD. Cut-off value at 0.680 has the highest sensitivity, specificity, positive predictive value and negative predictive value than others.

Table VI

\begin{tabular}{lcccc}
\hline $\begin{array}{l}\text { CIMT Cut- } \\
\text { off (mm) }\end{array}$ & Sensitivity & Specificity & PPV & NPV \\
\hline 0.610 & $77 \%$ & $55 \%$ & $64 \%$ & $69 \%$ \\
0.645 & $75 \%$ & $57 \%$ & $65 \%$ & $68 \%$ \\
$\mathbf{0 . 6 8 0}$ & $\mathbf{7 5 \%}$ & $\mathbf{6 3 \%}$ & $\mathbf{6 8 \%}$ & $\mathbf{7 0 \%}$ \\
0.775 & $50 \%$ & $87 \%$ & $81 \%$ & $62 \%$ \\
\hline
\end{tabular}

$\mathrm{PPV}=$ positive predictive value, $\mathrm{NPV}=$ negative predictive value

Table VII shows odds ratio analysis for the risk factors of CIMT $\geq 0.68 \mathrm{~mm}$. Risk factors for CIMT when $\geq 0.68 \mathrm{~mm}$ showed that only NAFLD had a risk for developing this condition $(\mathrm{P}<0.001)$. Gender, family history of CVD, DM and current smoking habit were not significant.

Table VII

\begin{tabular}{lcc}
\hline Variable & OR & $95 \%$ CI \\
& $($ CIMT $\geq 0.68$ \\
& $\mathrm{mm} /<0.68 \mathrm{~mm})$ \\
\hline
\end{tabular}

NAFLD

No

Yes

Male

Female

0.435

0.277

1

1.575

$0.553-4.485$

Family history of DM

$$
\text { Yes }
$$

No

Current smoking habit

$$
\text { No }
$$

0.377

1

0.800

$0.348-1.838$

$2.19-12.15$

$5.167 \quad 2.19-12.15$

1

0.863

$0.392-1.899$

9


Figure IV shows CIMT at cut-off point $1 \mathrm{~mm}$ in subjects with or without NAFLD. Total 14 (13.9\%) out of 101 subjects had more than $1 \mathrm{~mm}$ thickness of CIMT. Out of 14 subjects only $2 / 49$ (4.1\%) were having normal liver whereas 12/52 (23.1\%) had different grades of fatty liver disease. Considering CIMT cut-off at $1 \mathrm{~mm}$, fatty liver is 6 times more common $(\mathrm{p}<0.05)$.

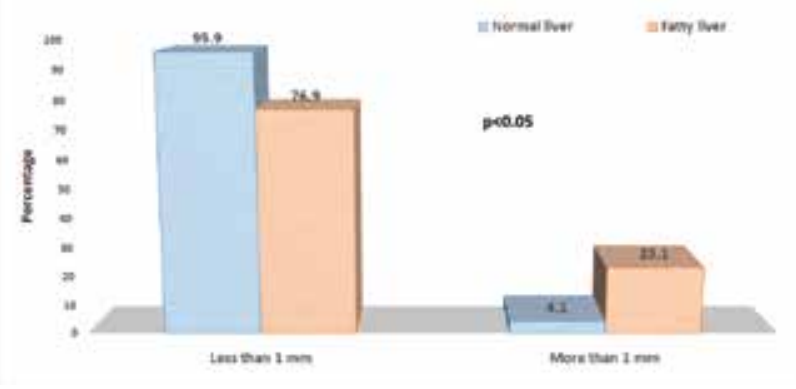

Figure IV

\section{Discussion}

The rising prevalence of NAFLD is related to the epidemic of obesity. Although the histologic picture resembles that of alcohol-induced liver injury, NAFLD occurs in patients who do not abuse alcohol. The histologic hallmark of NAFLD is predominantly macrovesicular steatosis. NAFLD is now increasingly being recognized as a cause of end-stage liver disease and is associated with increased rates of hepatocellular carcinoma, liver transplantation, and death.

The current study comprised of 101 patients who attended the outpatient department of Radiology \& Imaging, Dhaka Medical College. The mean age of the normal liver group was 36 years whereas it was in the NAFLD group 46 years. Mean age of the study subjects were significantly differed among two groups with or without NAFLD $(p<0.001)$ (Table I). Gender ( $p=0.233)$, family history of CVD $(p=0.599)$, family history of DM ( $\mathrm{p}=0.059)$ and current smoking habit $(\mathrm{p}=0.771)$ played no role in the statistical difference among two groups.

This study was designed to ascertain whether an incidental finding of NAFLD in outpatients may suggest the search for carotid artery intima thickness. The biological mechanisms of accelerated atherosclerosis contributed by NAFLD are still poorly understood. NAFLD itself might act as a stimulus for further increased whole-body insulin resistance and dyslipidemia, leading to accelerated atherosclerosis. Recent prospective studies demonstrated that presence of components of metabolic syndrome plays role in the development of fatty liver disease. ${ }^{23,24,25}$ Our results also showed that raised BMI and serum cholesterol which are components of metabolic syndrome (Mets) were significantly associated in the development of fatty liver disease $\mathrm{p}$ value $<0.001$ and 0.030 respectively (Table II).

On the other hand, systolic and diastolic blood pressure, fasting blood glucose, serum triglyceride levels were not
Figure V shows accuracy of the CIMT in patients with or without NAFLD by ROC curve. Accuracy of the CIMT (mm) in the diagnosis of NAFLD shown in the area under the curve (AUC) which is 0.752 and indicates fair outcome of the study.

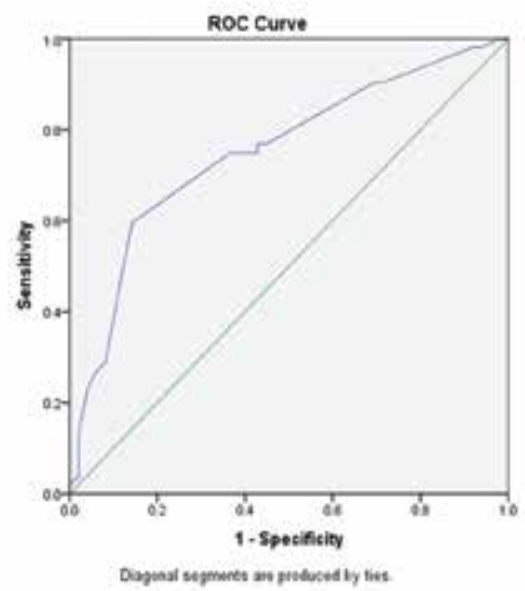

Figure V

statistically significant among normal liver and fatty liver groups. These findings are not concordant with previous result where several other studies showed that NAFLD is commonly associated with visceral obesity, dyslipidemia, insulin resistance, and type 2 diabetes. ${ }^{1,8,9}$ These phenomena could be explained by small number of study population in our study.

Growing age has a correlation with the increased severity of fatty liver disease in this study. The two extreme age groups i.e. $<30$ years and $>60$ years had a very few number of subjects enrolled both in normal liver and non NAFLD groups. Otherwise it was observed that the age has a good association (Fig II). Grade I fatty liver was more among young age groups whereas grade II fatty liver was higher by increasing age. This findings were similar to the study carried out by $\mathrm{Kim}^{26}$

Carotid intima media thickness as shown in figure 8 revealed that it increases with the severity of fatty liver. Mean CIMT in normal liver group was $0.62 \mathrm{~mm}$, grade I fatty liver $0.75 \mathrm{~mm}$, grade II fatty liver $0.76 \mathrm{~mm}$ and in grade III fatty liver 0.83 $\mathrm{mm}$. There is a linear correlation with these two conditions.

Our study was aimed to find out the association of NAFLD with CIMT. Before commencement of our study it was hypothesized that CIMT will not be higher in the fatty liver disease than that of normal subjects. At the end of the study the findings rejected this hypothesis. The results shown in Table III and Table IV had a significant value $(\mathrm{p}<0.001)$ among the normal liver (CIMT mm mean \pm SD, $0.62 \pm 0.15$ ) and the fatty liver subjects (CIMT mm mean \pm SD, $0.77 \pm 0.17$ ). Similar results were found by some case-controlled and cross-sectional studies ${ }^{10,11,12}$ and studies also showed a relationship between NAFLD and CIMT. ${ }^{11,15,19}$

However, some degree of variability about the mean CIMT values has been observed among all the published reports that result in a difficult evaluation of the magnitude of the 
observation. For instance, among the different studies, mean CIMT values in NAFLD patients range from $0.64 \pm 0.10 \mathrm{~mm}$ to $1.24 \pm 0.13 \mathrm{~mm}^{15,19}$ These results are very much concordant with our study. Targher et $\mathrm{al}^{10}$ also found that NAFLD was associated with a high cardiovascular risk and, in particular, an increased prevalence of carotid lesions.

In Fig IV, CIMT at cut-off point $1 \mathrm{~mm}$ was shown in subjects with or without NAFLD. Total $14(13.9 \%)$ out of 101 subjects had more than $1 \mathrm{~mm}$ thickness of CIMT. Out of 14 subjects only $2 / 49(4.1 \%)$ were having normal liver whereas $12 / 52$ $(23.1 \%)$ had different grades of fatty liver disease. Considering CIMT cut-off at $1 \mathrm{~mm}$, fatty liver is 6 times more common $(p<0.05)$. Currently available epidemiological data indicate that a value of CIMT at or above $1 \mathrm{~mm}$ at any age is associated with a significantly increased risk of myocardial infarction and/or cerebrovascular disease..$^{18}$

Considering gender discrimination between NAFLD and CIMT, the findings were highly significant among groups. For both male and female subjects the results were statically significant, $\mathrm{p}$ value among males was 0.001 and females was 0.010 (table V). Kim et $\mathrm{al}^{26}$ in a recent study found women with NAFLD had a higher C-IMT than those without NAFLD. Their finding meant that women with an upper normal ALT concentration might be susceptible to carotid atherosclerosis if they have NAFLD.

We compared the cut-off point of CIMT for the accuracy for diagnosing NAFLD, the value of $0.680 \mathrm{~mm}$ (Table VI) and the area under the curve (AUC) 0.752 (Fig V). This AUC value appered fair for the accuracy rate. Sensitivity, specificity, PPV and NPV at cut off value of $0.61 \mathrm{~mm}$ were 77 , 55, 64 and 69; at $0.64 \mathrm{~mm}$ were 75, 57, 65 and 68 ; at $0.68 \mathrm{~mm}$ were $75,63,68$ and 70 , at $0.77 \mathrm{~mm}$ were 50,87, 81 and 62 respectively (Table VI). Diagnostic accuracy of CIMT was observed more at cut off value $0.68 \mathrm{~mm}$. Similar results were found by some case-controlled and cross-sectional studies showed a relationship between NAFLD and CIMT. ${ }^{10,11,12,15,19}$

Risk factors for increased CIMT were analyzed where presence or absence of NAFLD only had statistically significant results $[\mathrm{p}<0.001$, odds ratio 5.167 and $95 \%$ CI (2.19-12.15)] (Table VII).. Lankarani et al. ${ }^{27}$ found, NAFLD patients had significantly higher prevalence of increased CIMT (OR, 1.66; $\mathrm{p}<0.001)$ compared to normal individuals.

In conclusion, this study was aimed to explore the association between non-alcoholic fatty liver disease and carotid artery intima-media thickness on B-mode ultrasonogram. There is a positive correlation between NAFLD with CIMT. High BMI and raised serum cholesterol has significant role in the development of NAFLD. Carotid screening for NAFLD might be beneficial for assessment of future atherosclerotic complications.

\section{Reference:}

1. Angulo P. Nonalcoholic Fatty Liver Disease. N Engl J Med. 2002; 16:1221-31.

2. Moller DE, Berger JP, Hui JM. Hepatic steatosis and insulin resistance. Pharmacol Ther 2005; 22:64-70.

3. El-Serag HB, Manson AC, Marrero JA. Stenosis, the metabolic syndrome and cancer. Aliment Pharmacol Ther. 2005 ;22:40-43.

4. Nanditha A, Ma RCW, Ramachandran A, Snehalatha C, Chan JCN et al. Diabetes in Asia and the Pacific: Implications for the Global Epidemic. Diabetes Care 2016; 39:472-485

5. Das SK, Mukherjee S, Vasudevan DM. Nonalcoholic Fatty liver disease: an under- recognized cause with emerging importance. Curr Sci 2006; 90:5.

6. Hernaez R, Lazo M, Bonekamp S, Kamel I, Brancati FL, Guallar E, Clark JM. Diagnostic accuracy and reliability of ultrasonography for the detection of fatty liver: a meta-analysis. Hepatology 2011; 54:1082-1090.

7. Duseja A, Singhy SP, Saraswatz VA, Acharya SK, Chawla YK. Non-alcoholic Fatty Liver Disease and Metabolic Syndrome-Position Paper of the Indian National Association for the Study of the Liver, Endocrine Society of India, Indian College of Cardiology and Indian Society of Gastroenterology. J Clin Exp Hepatol $2015 ; 5: 51-68$

8. Adams LA, Angulo P. Recent concepts in non-alcoholic fatty liver disease. Diabet Med 2005; 22:1129-33.

9. McCullough AJ. The clinical features, diagnosis and natural history of nonalcoholic fatty liver disease. Clin Liver Dis 2004; 8:521-33, viii.

10. Targher G, Bertolini L, Padovani R, Zenari L, Zoppini G, Falezza G. Relation of nonalcoholic hepatic steatosis to early carotid atherosclerosis in healthy men: role of visceral fat accumulation. Diabetes Care 2004; 27:2498-500.

11. Aygun C, Kocaman O, Sahin T, Uraz S, Eminler AT, Celebi A, Senturk O, Hulagu S. Evaluation of metabolic syndrome frequency and carotid artery intima-media thickness as risk factors for atherosclerosis in patients with nonalcoholic fatty liver disease. Dig Dis Sci $2008 ; 53: 1352-7$.

12. Targher G, Bertolini L, Rodella S, Tessari R, Zenari L, Lippi G, Arcaro G. Nonalcoholic fatty liver disease is independently associated with an increased incidence of cardiovascular events in type 2 diabetic patients. Diabetes Care 2007; 30:2119-21.

13. Musso G, Gambino R, Bo S, Uberti B, Biroli G, Pagano G, Cassader M. Should nonalcoholic fatty liver disease be included in the definition of metabolic syndrome? A cross-sectional comparison with Adult Treatment Panel III criteria in nonobese nondiabetic subjects. Diabetes Care 2008; 31:562-8.

14. Marchesini G, Brizi M, Bianchi G, Tomassetti S, Bugianesi E, Lenzi M, McCullough AJ, Natale S, Forlani G, Melchionda N. Nonalcoholic fatty liver disease: a feature of the metabolic syndrome. Diabetes 2001; 50:1844-50.

15. Kim HC, Kim DJ, Huh KB. Association between nonalcoholic fatty liver disease and carotid intima-media thickness according to the presence of metabolic syndrome. Atherosclerosis 2009; 204:521-5.

16. Jeong JW. Intima-media thickness of the carotid artery: non-invasive marker of atherosclerosis. J Korean Soc Echocardiogr $2002 ; 10: 8-12$

17. O’Leary DH, Polak JF, Kronmal RA, Manolio TA, Burke GL, Wolfson SK Jr. Carotid-artery intima and media thickness as a risk factor for myocardial infarction and stroke in older adults. Cardiovascular Health Study Collaborative Research Group. N Engl J Med 1999; 340:14-22. 
18. Park BH, Yoon GH, Park JH, Choi CS, Kook H, Yoo NJ, Oh SG, Jung JW, Park YG, Park OG. Relation of carotid artery intima-media thickness and atherosclerotic plaque with the extent of coronary artery stenosis. J Korean Soc Echocardiogr 2000; 8:45-53.

19. Volzke H, Robinson DM, Kleine V, Deutscher R, Hoffmann W, Ludemann J, Schminke U, Kessler C, John U. Hepatic steatosis is associated with an increased risk of carotid atherosclerosis. World J Gastroentero 2005; 11:1848-53.

20. Valls C, Iannacconne R, Alba E, Murakami T, Hori M, Passariello $\mathrm{R}$, et al . Fat in the liver: Diagnosis and characterization. Eur Radiol 2006; 16:2292-308.

21. Saadeh S, Younossi ZM, Remer EM, Gramlich T, Ong JP, Hurley M, et al. The utility of radiological imaging in nonalcoholic fatty liver disease. Gastroenterology 2002; 123:745-50.

22. Zhang L, Guo K, Lu J, Zhao F, Yu H, Han J et al. Nonalcoholic fatty liver disease is associated with increased carotid intima-media thickness in type 1 diabetic patients. Scientific Reports 2016; 6:26805. DOI: $10.1038 / \mathrm{srep} 26805$

23. Nannipieri M, Gonzales C, Baldi S, Posadas R, Williams K, Haffner SM, Stern MP, Ferrannini E; Mexico City diabetes study. Liver enzymes, the metabolic syndrome, and incident diabetes: the Mexico City diabetes study. Diabetes Care 2005; 28:1757-62.
24. Hanley AJ, Williams K, Festa A, Wagenknecht LE, D'Agostino RB Jr, Haffner SM. Liver markers and development of the metabolic syndrome: the insulin resistance atherosclerosis study. Diabetes 2005; 54:3140-7.

25. Chol SY, Kim D, Kang JH, Park MJ, Kian YS, Lim SH et (2008). Nonalcoholic fatty liver disease as a risk factor of cardiovascular disease; relation of non-alcoholic fatty liver disease to carotid atherosclerosis. The Korean J Hepatol 2008; 14:77-88

26. Kim KS, Oh HJ, Kim DJ, Kim SK, Park SW, Cho YW and Huh KB. The association between non-alcoholic fatty liver disease and carotid atherosclerosis in subjects with within-reference range alanine aminotransferase levels. Endocrine Journal 2013; 60(12), 1295-1301.

27. Lankarani KB, Mahmoodi M, Lotfi M, Zamiri N, Heydari ST, Ghaffarpasand F, et al. Common Carotid Intima-media Thickness in Patients with Non-alcoholic Fatty Liver Disease: A Population-based Case-control Study. Korean J Gastroenterol 2013; 62(6): 344-351. 